\title{
The benefits of trastuzumab in the treatment of HER2+ breast cancer as a function of exposure time
}

\author{
Felipe Andrés Cordero da Luz ${ }^{1,2 a}$ (iD, Eduarda da Costa Marinho ${ }^{1 \mathrm{~b}}$ (iD, Camila Piqui Nascimento ${ }^{1 \mathrm{c}}$ (iD, Lara de Andrade Marques ${ }^{1 \mathrm{~d}}$ (iD, \\ Patrícia Ferreira Ribeiro Delfino ${ }^{1 \mathrm{e}}$ (iD, Rafael Mathias Antonioli ${ }^{1 \mathrm{f}}$ (iD, Marcelo José Barbosa Silva ${ }^{2 \mathrm{~g}}$ (iD) and Rogério Agenor de Araújo ${ }^{1,3 \mathrm{~h}}$
}

${ }^{1}$ Center for Cancer Prevention and Research, Uberlandia Cancer Hospital, Av Amazonas n 1996, Umuarama, Uberlândia, Minas Gerais, 38.405-302, Brazil ${ }^{2}$ Laboratory of Tumor Biomarkers and Osteoimmunology, Institute of Biomedical Sciences, Federal University of Uberlandia, Av Pará $n^{\circ} 1720$, Bloco 6T, room 07, Umuarama, Uberlândia, Minas Gerais, 38.405-320, Brazil

${ }^{3}$ Faculty of Medicine, Federal University of Uberlandia, Av Pará n 1720, Bloco 6T2u, room 07, Umuarama, Uberlândia, Minas Gerais, 38.400-902, Brazil ahttps://orcid.org/0000-0002-9381-4913

bhttps://orcid.org/0000-0002-1307-9104

chttps://orcid.org/0000-0002-0955-8559

dhttps://orcid.org/0000-0002-2734-8352

ehttps://orcid.org/0000-0002-2196-9318

fhttps://orcid.org/0000-0003-3886-1562

ghttps://orcid.org/0000-0002-5807-4286

hhttps://orcid.org/0000-0003-4653-6786

\section{Abstract}

Background: Breast cancer is a heterogeneous disease with overexpression of several receptors, such as human epidermal receptor 2 (HER2), which is a prognostic and predictive biomarker for treatment with the anti-HER2 monoclonal antibody trastuzumab. This study aimed to test the contribution of this regimen in patients with overexpression/ amplification of HER2 for periods shorter than the 1-year treatment recommendation.

Methods: A retrospective single-centre study involving 155 patients with non-metastatic (stages I-III) invasive ductal HER2+ breast carcinoma, with a median follow-up of 48.9 months after completion of adjuvant therapy, except endocrine therapy.

Results: About $60 \%$ of patients received trastuzumab therapy for a median time of 365 days. Although the use of trastuzumab for a short period has provided some benefit, analyses of survival with a continuous dependent variable have revealed a minimum time for improved survival. In the multivariate analysis by Cox regression, trastuzumab use duration exceeding 9 weeks resulted in protection against distant metastasis (adjusted HR: 0.307 (0.139-0.678), $p=0.004$ ), disease progression (adjusted hazard ratio (HR) 0.353 (0.175-0.714), $p=0.004$ ) and death (adjusted HR: 0.267 (0.105-0.678), $p=0.005)$, being superior to multimodal systemic therapy with chemotherapy and to endocrine therapy without trastuzumab, but inferior to almost 1 year of administration of this monoclonal antibody, especially regarding overall survival (adjusted HR: 0.203 (0.069-0.596), $p=0.004)$.

Conclusion: Despite showing some benefits, the protective effect derived from a suboptimal time of trastuzumab exposure is inferior to the standard course of 1 year.

Keywords: antineoplastic agents, breast neoplasms, ErbB-2 receptor, prognosis, trastuzumab

Correspondence to: Felipe Andrés Cordero da Luz Email: felipecorderodaluz@gmail.com

ecancer 2022, 16:1347

https://doi.org/10.3332/ecancer.2022.1347

Published: $20 / 01 / 2022$

Received: 27/07/2021

Publication costs for this article were supported by ecancer (UK Charity number 1176307).

Copyright: (C) the authors; licensee ecancermedicalscience. This is an Open Access article distributed under the terms of the Creative Commons Attribution License (http:// creativecommons.org/licenses/by/4.0), which permits unrestricted use, distribution, and reproduction in any medium, provided the original work is properly cited. 


\section{Background}

Breast cancer is a highly heterogeneous disease, classified by the TNM ( $\mathrm{T}$ - tumour; $\mathrm{N}$ - lymph node metastasis; $\mathrm{M}$ - distant metastasis) system, histological grade and expression of hormone receptors (oestrogen - ER and progesterone - PR) and human epidermal receptor 2 (HER2) [1-4]. The overexpression (intense membrane staining of $>10 \%$ of tumour cells by immunohistochemistry) and/or amplification of the ERBB2 gene locus, which codes for HER2, is necessary for classification as HER2+ tumours [5].

Irrespective of the expression of hormone receptors, $15 \%-20 \%$ of breast cancers are of the HER2+ subtype [3, 6]. Historically, HER2 overexpression/amplification is related to the worst outcome in breast cancer patients [7]. However, the development of targeted trastuzumab therapy has caused a revolution in the prognosis of these patients [8]. Trastuzumab is a neutralising monoclonal antibody that mainly leads to HER2 blockade, by impeding its signalling pathway activation, which is associated with cell proliferation and survival [9]. Thus, its amplification/overexpression leads to rapid tumour progression [9]. This is corroborated by the fact that HER2 prevails as a worse prognostic factor in patients who did not receive adequate systemic therapy [10].

The availability of this therapy caused such a revolution that it led to the overexpression/amplification of HER2 as a downstaging criterion in the recent staging manual (eighth edition) by the American Joint Committee on Cancer (AJCC) [11, 12]. However, its high cost over an entire 1-year course of treatment [9] hinders both its implementation and its continuous supply in public health services of developing countries, such as Brazil $[13,14]$. Thus, the search for a shorter time of exposure to trastuzumab, with equivalent benefits, is essential to be able to offer this indispensable therapy uninterruptedly and universally to all these women, so that they do not suffer the deleterious effect that the amplification/overexpression of HER2 has on their prognosis [9, 14]. In this regard, despite some contradictions, some clinical trials have demonstrated that the benefit of a shorter regimen with trastuzumab did not lose effectiveness [9], generating potential savings and availability to a greater number of patients [14].

This retrospective study aimed to analyse the benefit of trastuzumab in the treatment of patients with breast cancer undergoing adjuvant treatment attended in a public health unit in Brazil, and test whether there is a minimum exposure time, less than 1 year, which generates protection.

\section{Methods}

\section{Study design}

A retrospective observational study of patients with breast cancer treated at the oncology sector of the Federal University of Uberlandia between January 1999 and November 2021 was performed. Based on the result of the anatomopathological examination, and not on the medical notes, all patients were reclassified in their pathological TNM according to the Seventh Edition of the AJCC [15]. For prognostic analysis, both $\mathrm{T}$ and $\mathrm{N}$ classifications were obtained by clinical and pathological examinations, independently if the patient received neoadjuvant therapy. Patients' ages were classified into two categories according to the following criteria: $<70$ years and $\geq 70$ years for overall survival (OS) due to shorter life expectancy, higher risk of all-cause death and having received fewer treatments [16, 17].

The systemic treatment was considered adequate whenever patients received treatment as indicated by current guidelines. A systemic treatment was considered correct when patients received complete course of chemotherapy, according to chemotherapy schema, and trastuzumab (except when $\mathrm{T}<1 \mathrm{~cm}-\mathrm{T} 1 \mathrm{a} / \mathrm{T} 1 \mathrm{~b}$ ), for a minimum time of 1 year, and received endocrine therapy when tumours expressed at least one positive hormone receptor [1,2]; hormone receptor expression was considered positive when at least $1 \%$ of tumour cells were detected as expressing by the immunohistochemistry approach [4]. Trastuzumab exposure/administration time was measured in days between the date of the first dose and the date of the last dose but the exposure to at least 14 cycles of trastuzumab was also considered adequate.

\section{Ethical factor}

This study was approved by the Human Research Ethics Committee of the local Institution (protocol number 803.826/14) and followed all the ethical principles of the Declaration of Helsinki and its subsequent amendments or comparable ethical standards. The informed consent form was waived, according to the type of study performed. 


\section{Inclusion and exclusion criteria}

This study enrolled female patients with invasive ductal carcinoma of no special type/no otherwise specified histology with overexpression/ amplification of HER2. Patients were excluded according to the following criteria: synchronic metastatic disease (initial diagnosis or within 6 months); missing histopathological data (surgical margin or histological grade); incomplete immunohistochemistry (absence of ER or PR) and/ or indeterminate HER2 (2+ with indeterminate/without hybridisation method); failure to perform surgery; neoadjuvant radiation therapy; the presence of a special component (papillary, mucinous, cribriform, etc.); lysed/destroyed tumour; bilateral cancer; more than one primary cancer; tumour progression during adjuvant treatment, except endocrine therapy; unreported cause of death and follow-up time less than 180 days from diagnosis to event.

From a total of 2,580 medical records, 1,685 were excluded for being metastatic or in situ tumours, or lack of adequate pathological examination, lack of surgery, neoadjuvant radiation therapy, development of another cancer and/or bilateral/contralateral breast cancer. Of the remaining 895 medical records, 737 were excluded for being Luminal A or B or TN. Three patients were subsequently excluded due to tumour progression during chemotherapy, trastuzumab or radiation therapy course.

\section{Outcomes}

Distant metastasis-free survival (DMFS) was defined as the time from diagnosis to development of contralateral lymph node metastasis and/ or to any distant organ. Disease-free survival (DFS) was considered the time from diagnosis to development of any relapse (local, regional or distant metastasis) or death from any cause. OS was defined as the time from diagnosis to death from any cause.

\section{Statistical analysis}

The Kolmogorov-Smirnov normality test, descriptive analyses, cross-table analysis of concordance (Cohen's Kappa), Kaplan-Meier and Cox regression were performed on the software IBM SPSS v25.0. The survival curves with continuous predictor and their optimal cutoff point were established using the software Jamovi v1.6.5.0. In all analyses, statistical significance was defined as $p<0.05$.

The Kaplan-Meier estimator was used to analyse the proportionality of risks as a prerequisite for considering the variable in the Cox regression model in multivariate analysis. The time-dependent Cox regression model was used to test the influence of time from diagnosis to the end of adjuvant therapy, except endocrine therapy, on outcomes. Multivariate Cox regression was performed using the Stepwise Forward Wald method with an entry $p$-value of 0.25 [18] and output $p$-value of 0.10 for analysis of independent prognosis factor with superiority.

\section{Results}

Of the included patients ( $n=155)$, only $96(61.9 \%)$ received trastuzumab, for a median time of 365 days, but only $70(45.2 \%)$ received an adequate treatment according to the 2007 St. Gallen Consensus standard guidelines [19]. All characteristics are depicted in Table 1.

\section{Trastuzumab treatment has a positive impact on a patient's prognosis}

The added benefit of trastuzumab treatment was later tested in relation to the endpoints of distant metastasis, any progression and death.

First, it was tested whether the time from diagnosis to end of therapy could imply immortal time bias. By time-dependent Cox regression, this time had a non-significant impact only on disease progression (hazard ratio (HR): $150.357(0.738-30,616.233), p=0.065)$. Due to this result and theoretical background (unpublished results), only the time from the end of adjuvant (surgery, chemotherapy, trastuzumab or radiation therapy) to the observed outcome was further considered.

Then, it was tested whether the duration of trastuzumab exposure could impact outcomes. By continuous survival analysis, it was observed that the duration of trastuzumab exposure has a significant impact on DMFS (HR: 1.00 (0.99-1.00), $p=0.011)$ and OS (HR: 1.00 (0.99-1.00), $p=0.008$ ) and is inversely associated with the outcome when there were more than 292 days of trastuzumab exposure. On the other hand, 
although also significant for DFS (HR: $1.00(1.00-1.00), p=0.018)$, it was inversely associated with the outcome when there were more than 63 days of trastuzumab exposure. Kaplan-Meier plots are depicted in Figures 1-3. The median time of survival (50\%) was achieved only for patients with up to 63 days/no exposure to Trastuzumab regarding DFS (133.23 months).

Because of these discrepancies, patients were categorised according to time of Trastuzumab administration: 1) $\leq 63$ versus $>63$ days (9 weeks) of exposure and 2) $\leq 292$ versus $>292$ days of exposure. Additionally, it was tested whether any used time of exposure - either more than 84 days (12 weeks) or at least 6 months of exposure - could provide some benefit. An exposure of fewer than 292 days did not differ significantly from no exposure at all (Figures 1 and 3 ). The patients with no trastuzumab exposure were clustered together with patients receiving suboptimal time of trastuzumab exposure.

Based on these cutoffs, 93 patients (60.0\%) were classified as receiving trastuzumab for more than 63 days, 91 (58.7\%) patients as receiving it for 12 weeks or more, $86(55.5 \%)$ as receiving this treatment for 6 months or more and $82(52.9 \%)$ as receiving it for more than 292 days. Their counterparts included 62 (59 no treatment and 3 with less than or equal to 63 days of exposure), 64 ( 59 no treatment and 5 a maximum of 12 weeks of exposure), 69 (59 no treatment and 11 with less than 6 months of exposure) and 73 (59 no treatment and 15 with less than or equal to 292 days of exposure) patients, respectively.

Table 1. Clinical data of included patients $(n=155)$.

\begin{tabular}{|l|c|c|}
\hline \multicolumn{1}{|c|}{ Variable } & $N(\%)$ & $\begin{array}{c}\text { Median (minimum- } \\
\text { maximum)/mean ( } \pm \text { SD) }\end{array}$ \\
\hline Time to metastasis & $155(100)$ & 61.87 months (7.00-184.80) \\
\hline Time to progression & $155(100)$ & 60.07 months (7.00-184.80) \\
\hline Time to death & $155(100)$ & 65.83 months (7.00-184.80) \\
\hline & & \\
\hline Time to end of adjuvance & $155(100)$ & 16.93 months (1.40-53.40) \\
\hline Age & $155(100)$ & 53 years (28-79) \\
\hline Age & & \\
\hline$<70$ years & $146(94.2)$ & \\
\hline$\geq 70$ years & $9(5.8)$ & \\
\hline Distant metastasis developed & & \\
\hline No & $126(81.3)$ & \\
\hline Yes & $29(18.7)$ & \\
\hline Progression & & \\
\hline No & $119(76.8)$ & \\
\hline Yes & $36(23.20)$ & \\
\hline Deaths & & \\
\hline No & & \\
\hline Yes & & \\
\hline & & \\
\hline & & \\
\hline T (initial) & & \\
\hline T1 & & \\
\hline & & \\
\hline & & \\
\hline & & \\
\hline
\end{tabular}


Table 1. Clinical data of included patients $(n=155)$. (Continued)

\begin{tabular}{|c|c|c|}
\hline $\mathrm{T} 2$ & $63(40.6)$ & \\
\hline T3 & $30(19.4)$ & \\
\hline T4 & 25 (16.1) & \\
\hline \multicolumn{3}{|l|}{$N$ (initial) } \\
\hline No & $68(43.9)$ & \\
\hline N1 & $48(31.0)$ & \\
\hline N2 & $26(16.8)$ & \\
\hline N3 & $13(8.4)$ & \\
\hline \multicolumn{3}{|l|}{$\mathrm{T}$ (pathological) } \\
\hline T1 & $55(35.5)$ & \\
\hline $\mathrm{T} 2$ & $77(49.7)$ & \\
\hline T3 & $13(8.4)$ & \\
\hline T4 & $10(6.5)$ & \\
\hline \multicolumn{3}{|l|}{$\mathrm{N}$ (pathological) } \\
\hline NO & $84(54.2)$ & \\
\hline N1 & $38(24.5)$ & \\
\hline N2 & $23(14.8)$ & \\
\hline N3 & $10(6.5)$ & \\
\hline \multicolumn{3}{|l|}{ Histological grade } \\
\hline G1 & $6(3.9)$ & \\
\hline G2 & $106(68.4)$ & \\
\hline G3 & $43(27.7)$ & \\
\hline \multicolumn{3}{|l|}{ Hormone receptor } \\
\hline Negative & $64(41.3)$ & \\
\hline Positive & $91(58.7)$ & \\
\hline \multicolumn{3}{|c|}{ Trastuzumab (any quantity) } \\
\hline Did not received & $59(38.1)$ & \\
\hline Received & $96(61.9)$ & 365 days $(21-502)$ \\
\hline \multicolumn{3}{|c|}{ Trastuzumab (63 days) } \\
\hline$\leq 63$ days & $3(3.1)$ & 63 days (21-63) \\
\hline$>63$ days & $93(96.9)$ & 365 days $(71-502)$ \\
\hline \multicolumn{3}{|c|}{ Trastuzumab (12 weeks) } \\
\hline$\leq 12$ weeks & $5(5.2)$ & 63 days (21-82) \\
\hline$>12$ weeks & $91(94.89)$ & 365 days (126-502) \\
\hline \multicolumn{3}{|c|}{ Trastuzumab (6 months) } \\
\hline$<6$ months & $10(10.4)$ & 104 days $(21-169)$ \\
\hline$\geq 6$ months & $86(89.6)$ & 367 days (216-502) \\
\hline
\end{tabular}


Table 1. Clinical data of included patients $(n=155)$. (Continued)

\begin{tabular}{|l|c|l|}
\hline Trastuzumab (292 days) & & \\
\hline$\leq 292$ days & $59(38.1)$ & 142.5 days (21-292) \\
\hline$>292$ days & $96(61.9)$ & 369 days (296-502) \\
\hline Endocrine therapy & & \\
\hline Negative & $8 / 91(8.8)$ & \\
\hline Positive & $83 / 91(91.2)$ & \\
\hline Chemotherapy & & \\
\hline No & $10(6.5)$ & \\
\hline Neoadjuvant & $50(32.3)$ & \\
\hline Adjuvant & $95(61.2)$ & \\
\hline Systemic treatment (St. Gallen) & & \\
\hline Inadequate & $85(54.8)$ & \\
\hline Adequate & $70(45.2)$ & \\
\hline Locoregional treatment & & \\
\hline Inadequate & $14(9.0)$ & \\
\hline Adequate & $141(91.0)$ & \\
\hline
\end{tabular}

G1, Well differentiated; G2, Moderately differentiated; G3, Poorly differentiated;

$\mathrm{N}$, Lymph node metastasis; T, Tumour size
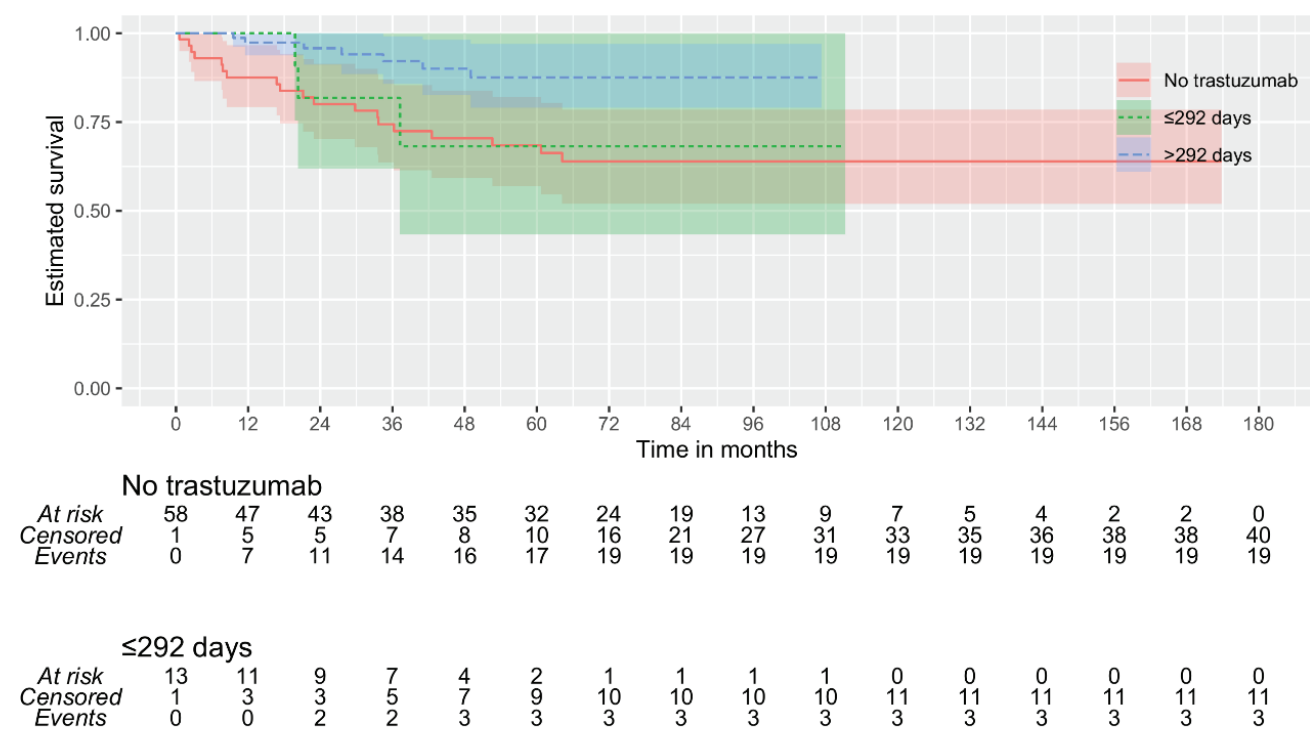

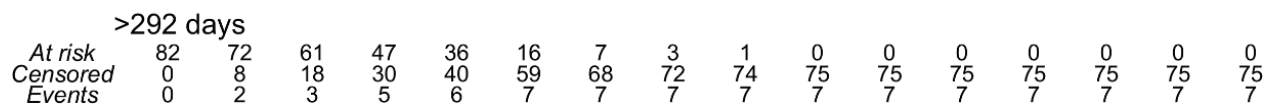

Figure 1. KMunicate plot for DMFS according to the time of administration of trastuzumab. Patients of the group of $\leq 292$ days of exposition included 59 of no treatment and 15 with less or equal to 292 days of the exposition. 


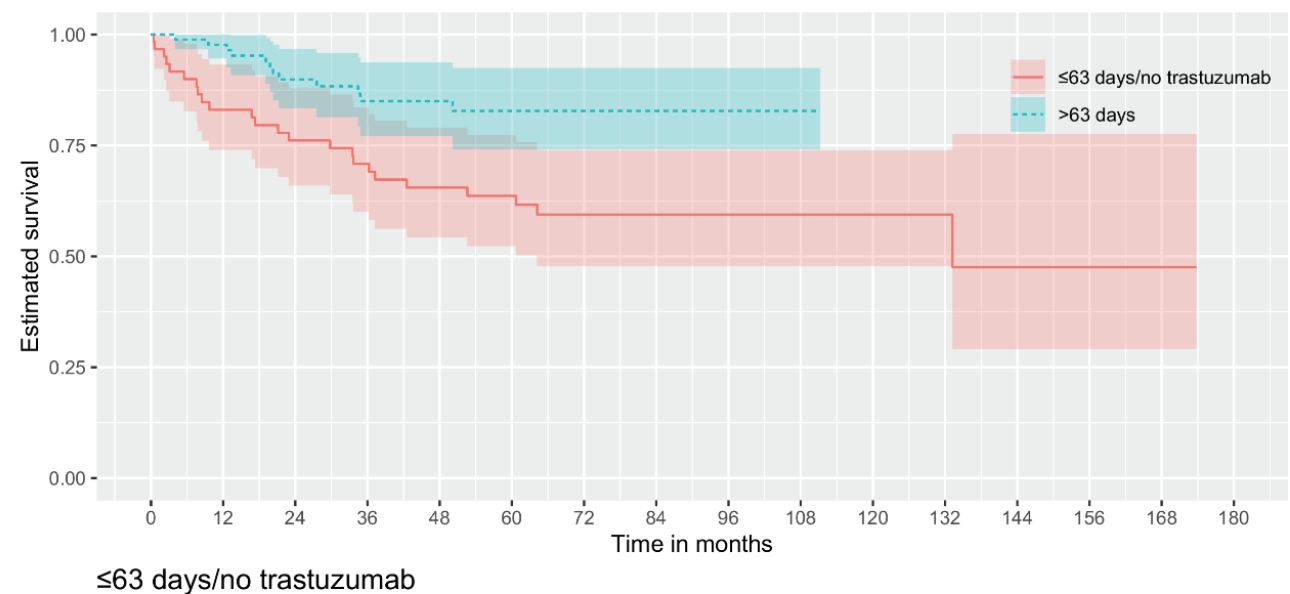

\begin{tabular}{|c|c|c|c|c|c|c|c|c|c|c|c|c|c|c|c|c|}
\hline At risk & 61 & 48 & 44 & 40 & 35 & 32 & 24 & 19 & 13 & 9 & 7 & 5 & 3 & 1 & 1 & 0 \\
\hline Censored & 1 & 4 & 4 & 5 & 7 & 9 & 15 & 20 & 26 & 30 & 32 & 34 & 35 & 37 & 37 & 38 \\
\hline Events & 0 & 10 & 14 & 17 & 20 & 21 & 23 & 23 & 23 & 23 & 23 & 23 & 24 & 24 & 24 & 24 \\
\hline
\end{tabular}

\begin{tabular}{|c|c|c|c|c|c|c|c|c|c|c|c|c|c|c|c|c|}
\hline & & & & & & & & & & & & & & & & \\
\hline At risk & 92 & 81 & 66 & 49 & 39 & 17 & 8 & 4 & 2 & 1 & 0 & 0 & 0 & 0 & 0 & 0 \\
\hline Censored & 1 & 10 & 19 & 33 & 43 & 64 & 73 & 77 & 79 & 80 & 81 & 81 & 81 & 81 & 81 & 81 \\
\hline Events & 0 & 2 & 8 & 11 & 11 & 12 & 12 & 12 & 12 & 12 & 12 & 12 & 12 & 12 & 12 & 12 \\
\hline
\end{tabular}

Figure 2. KMunicate plot for DFS according to the time of administration of trastuzumab. Patients of the group of $\leq 63$ days of exposition included 59 of no treatment and 3 with less or equal to 63 days of the exposition.

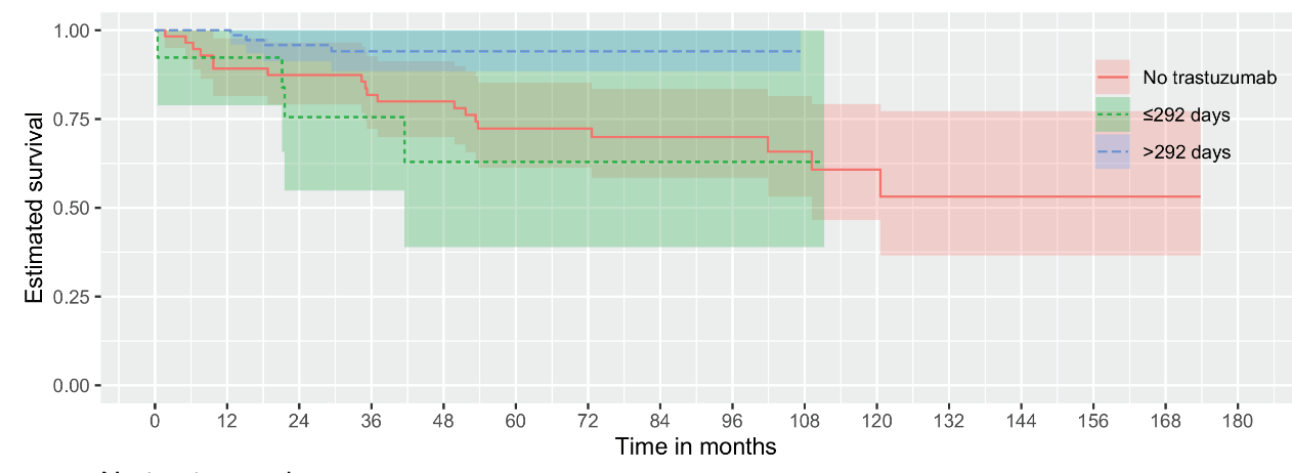

\begin{tabular}{ccccccccccccccccc}
\multicolumn{1}{c}{ No trastuzumab } \\
At risk & 58 & 49 & 48 & 44 & 42 & 36 & 30 & 24 & 18 & 13 & 8 & 5 & 4 & 2 & 2 & 0 \\
Censored & 1 & 4 & 4 & 5 & 6 & 8 & 14 & 19 & 25 & 29 & 33 & 35 & 36 & 38 & 38 & 40 \\
Events & 0 & 6 & 7 & 10 & 11 & 15 & 15 & 16 & 16 & 17 & 18 & 19 & 19 & 19 & 19 & 19
\end{tabular}
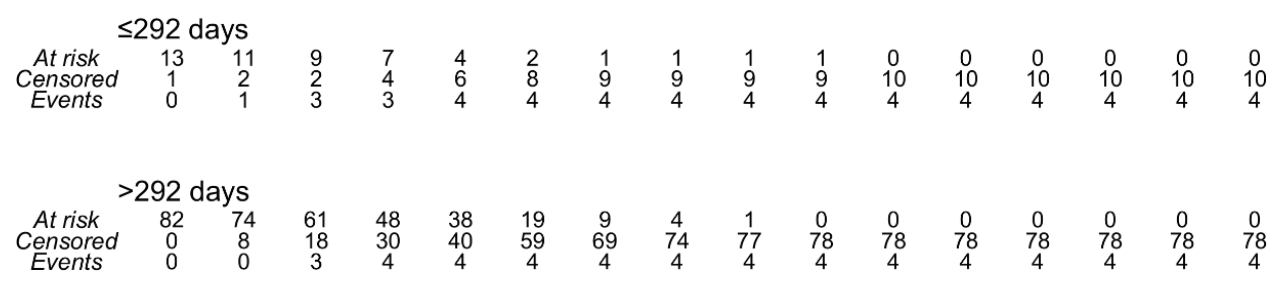

Figure 3. KMunicate plot for OS according to the time of administration of trastuzumab. Patients of the group of $\leq 292$ days of exposition included 59 of no treatment and 15 with less or equal to 292 days of the exposition. 
By stepwise analysis, the trastuzumab exposure exceeding 292 days resulted in increased capacity for protecting against distant metastasis (Table 2), and death (Table 3), and any progression. Furthermore, it was observed that the trastuzumab exposure for a longer time (>292 days) has a stronger impact than the multimodal treatments by the St. Gallen Consensus (Tables 2-4), though a moderate concordance was observed between trastuzumab administration and adequate treatment (Cohen's Kappa: 0.795, $p<0.0005$ ).

The trastuzumab exposure time obtained as optimal was very close to that recommended as standard (1 year), except for DFS. In order to test whether a shorter period offers similar protection, analyses were redone, but without inserting the optimal cutoff of each outcome in the model. This time, exposure for more than 63 days resulted in greater protection in stepwise multivariate Cox regression for DMFS (adjusted HR: 0.307 (0.139-0.678), $p=0.004$ ) and for OS (adjusted HR: $0.267(0.105-0.678), p=0.005)$, while an exposure exceeding 292 days was associated with improved DFS (adjusted HR: $0.358(0.172-0.748), p=0.006)$.

The former consensus preconised trastuzumab except in patients with T1a/T1b, NO disease [19]. Therefore, we excluded these patients from analysis $(n=8)$. Continuous survival analysis showed different cutoffs, of $>63$ days for DMFS $(p=0.0005)$ and DFS $(p=0.007)$, and of $>292$ days for OS $(p=0.0004)$.

Table 2. Univariate and multivariate Cox regression for DMFS of patients with HER2 tumours $(n=155)$.

\begin{tabular}{|c|c|c|c|c|}
\hline \multirow[b]{2}{*}{ Factor } & \multicolumn{2}{|l|}{ Univariate } & \multicolumn{2}{|c|}{ Multivariate } \\
\hline & $\mathrm{HR}(95 \% \mathrm{Cl})$ & $p$ & $\mathrm{HR}(95 \% \mathrm{Cl})$ & $p$ \\
\hline \multicolumn{5}{|c|}{ Trastuzumab exposition } \\
\hline No & 1 & & & \\
\hline Yes & $0.362(0.167-0.784)$ & 0.010 & & \\
\hline \multicolumn{5}{|c|}{ Trastuzumab exposition } \\
\hline$\leq 292$ days/no & 1 & & 1 & \\
\hline$>292$ days & $0.297(0.127-0.698)$ & 0.005 & $0.284(0.121-0.668)$ & 0.004 \\
\hline \multicolumn{5}{|c|}{ Trastuzumab exposition } \\
\hline$<6$ months/no & 1 & & & \\
\hline$\geq 6$ months & $0.333(0.147-0.756)$ & 0.009 & & \\
\hline \multicolumn{5}{|c|}{ Trastuzumab exposition } \\
\hline$<12$ weeks/no & 1 & & & \\
\hline$\geq 12$ weeks & $0.349(0.158-0.770)$ & 0.009 & & \\
\hline \multicolumn{5}{|c|}{ Trastuzumab exposition } \\
\hline$\leq 9$ weeks/no & 1 & & & \\
\hline$>9$ weeks & $0.329(0.149-0.726)$ & 0.006 & & \\
\hline \multicolumn{5}{|c|}{ St. Gallen Consensus treatment } \\
\hline Inadequate & 1 & & & \\
\hline Adequate & $0.503(0.222-1.137)$ & 0.099 & & \\
\hline \multicolumn{5}{|l|}{$\mathrm{pN}$} \\
\hline $\mathrm{N}-$ & 1 & & 1 & \\
\hline $\mathrm{N}+$ & $2.226(1.061-4.671)$ & 0.034 & $2.370(1.128-4.981)$ & 0.023 \\
\hline
\end{tabular}


Table 3. Univariate and multivariate Cox regression for OS of patients with HER2 tumours $(n=155)$.

\begin{tabular}{|c|c|c|c|c|}
\hline \multirow[b]{2}{*}{ Factor } & \multicolumn{2}{|l|}{ Univariate } & \multicolumn{2}{|c|}{ Multivariate } \\
\hline & $\mathrm{HR}(95 \% \mathrm{Cl})$ & $p$ & $\mathrm{HR}(95 \% \mathrm{Cl})$ & $p$ \\
\hline \multicolumn{5}{|l|}{ Trastuzumab exposition } \\
\hline No & 1 & & & \\
\hline Yes & $0.375(0.160-0.882)$ & 0.025 & & \\
\hline \multicolumn{5}{|c|}{ Trastuzumab exposition } \\
\hline$\leq 292$ days/no & 1 & & 1 & \\
\hline$>292$ days & $0.202(0.069-0.594)$ & 0.004 & $0.203(0.069-0.596)$ & 0.004 \\
\hline \multicolumn{5}{|l|}{ Trastuzumab exposition } \\
\hline$<6$ months/no & 1 & & & \\
\hline$\geq 6$ months & $0.249(0.092-0.670)$ & 0.006 & & \\
\hline \multicolumn{5}{|c|}{ Trastuzumab exposition } \\
\hline$<12$ weeks/no & 1 & & & \\
\hline$\geq 12$ weeks & $0.279(0.110-0.705)$ & 0.007 & & \\
\hline \multicolumn{5}{|l|}{ Trastuzumab exposition } \\
\hline$\leq 9$ weeks/no & 1 & & & \\
\hline$>9$ weeks & $0.264(0.104-0.668)$ & 0.005 & & \\
\hline \multicolumn{5}{|c|}{ St. Gallen Consensus treatment } \\
\hline Inadequate & 1 & & & \\
\hline Adequate & $0.278(0.095-0.815)$ & 0.020 & & \\
\hline \multicolumn{5}{|l|}{$\mathrm{pN}$} \\
\hline N0/N1 & 1 & & 1 & \\
\hline N2/N3 & $2.555(1.166-5.595)$ & 0.019 & $2.530(1.155-5.538)$ & 0.020 \\
\hline
\end{tabular}

Table 4. Univariate and multivariate Cox regression for DFS of patients with HER2 tumours $(n=155)$.

\begin{tabular}{|l|c|c|c|c|}
\hline \multicolumn{1}{|c|}{ Factor } & \multicolumn{2}{c|}{ Univariate } & \multicolumn{2}{c|}{ Multivariate } \\
\hline Trastuzumab exposition & HR $(95 \% \mathrm{Cl})$ & $p$ & HR (95\% Cl) & $p$ \\
\hline No & & & & \\
\hline Yes & 1 & & & \\
\hline Trastuzumab exposition & $0.451(0.228-0.891)$ & 0.022 & & \\
\hline$\leq 292$ days/no & 1 & & & \\
\hline$>292$ days & 1 & & & \\
\hline Trastuzumab exposition & $0.372(0.178-0.778)$ & 0.009 & & \\
\hline$<6$ months/no & & & & \\
\hline$\geq 6$ months & 1 & & & \\
\hline Trastuzumab exposition & $0.396(0.193-0.811)$ & 0.011 & & \\
\hline
\end{tabular}


Table 4. Univariate and multivariate Cox regression for DFS of patients with HER2 tumours $(n=155)$. (Continued)

\begin{tabular}{|l|c|c|c|c|}
\hline$<12$ weeks/no & 1 & & & \\
\hline$\geq 12$ weeks & $0.398(0.197-0.804)$ & 0.010 & & \\
\hline Trastuzumab exposition & & & & \\
\hline$\leq 9$ weeks/no & 1 & & 1 & \\
\hline$>9$ weeks & $0.375(0.186-0.757)$ & 0.006 & $0.353(0.175-0.714)$ & 0.004 \\
\hline St. Gallen Consensus treatment & & & & \\
\hline Inadequate & 1 & & & \\
\hline Adequate & $0.448(0.210-0.959)$ & 0.039 & & \\
\hline$p N$ & & & & \\
\hline $\mathrm{N}-$ & 1 & & & \\
\hline $\mathrm{N}+$ & $2.018(1.042-3.908)$ & 0.037 & $2.183(1.124-4.237)$ & 0.021 \\
\hline
\end{tabular}

DMFS, as the only cutoff point that differed, was tested for its ability to impact the multivariate analysis. By stepwise Cox regression, the cutoff based on 9 weeks of exposure was retained in the model, with improved DMFS (adjusted HR: $0.287(0.130-0.638), p=0.002$ ), and was not substantially different from the benefit associated with >292 days of exposure (adjusted HR: $0.269(0.114-0.634), p=0.003)$.

As the analyses included patients with no exposure to trastuzumab, analyses were performed again excluding these patients. No impact was observed regarding DMFS (HR: 1.00 (0.99-1.00), $p=0.565)$ nor DFS (HR: $1.00(0.99-1.00), p=0.422$ ), but OS was found to be inversely associated with an exposure greater than 292 days (HR: $0.99(0.99-1.00), p=0.042)$. The exposure time of $>292$ days resulted in improved OS (adjusted HR: $0.162(0.040-0.652), p=0.010)$ by multivariate Cox analysis.

\section{Discussion}

Trastuzumab is a turning point in treating breast cancer patients with HER2 overexpression/amplification [8]. Its implementation leads to improved DMFS [20], DFS [8, 20], OS [8] and even locoregional relapse-free survival [20]. This retrospective study demonstrated that trastuzumab is the leading systemic therapy associated with improved outcomes, even compared to an adequate multimodal systemic treatment.

In Brazil, the implementation of trastuzumab in the treatment guidelines of the public system only took place in 2013 [21]. Even before this occurred, discussion of the economic impact of this therapy in the recommended complete 1-year course suggested the implementation of shorter times [14]. In fact, the implementation of trastuzumab can increase the cost of treating a patient by approximately 300\% [13]. Additionally, even with a progressive decrease in the cost of trastuzumab, the augmented number of cases makes this therapy increase the economic burden of treating neoplasias [22]. Thus, searching for shorter optimal times of administration is critical. We observed that an exposure time greater than 63 days is associated with improved outcomes, especially those associated with relapses (DMFS and DFS).

Several clinical trials reported non-inferiority of a shorter time of trastuzumab administration compared to 12 months, such as 6 months [23], 12 weeks [24] and even 9 weeks [25,26]. Although one clinical trial failed to observe non-inferiority of 6 months compared to 1 year of trastuzumab administration [27], a recent meta-analysis reported non-inferior protection of 6 months compared to 1 year [28]. Interestingly, the optimal cutoff observed in this study was of 9 weeks ( 63 days). This cutoff provided protection against disease progression superior to 12 weeks and 6 months, suggesting that patients with an exposure between 9 weeks and 12 weeks or 6 months still derived benefit from trastuzumab exposure. This effect was more evident after excluding T1a/T1b patients, which did not indicate trastuzumab treatment in the previous guidelines [19]. Indeed, further studies have shown the benefit of adding trastuzumab in patients with T1b $(>0.5$ to $\leq 1 \mathrm{~cm})$-NO tumours [1], which can be considered a study bias that was also eliminated.

A shorter time of trastuzumab exposure could result in less toxicity but also an increased risk of disease progression [28]. Because the high number of patients who did not receive trastuzumab were grouped together with a small number who received suboptimal treatment, it is 
difficult to draw any firm conclusions about this. In order to elucidate the potential benefit of these suboptimal treatments, the study design included patients with increasing exposure times, either by cutoff points obtained in our analyses or by data from the literature. In this regard, there was statistical superiority in this type of analysis (Stepwise method) compared to simply any exposure time or no exposure, suggesting that an exposure time of $>9$ weeks can achieve significant protection against disease progression. However, we observed a benefit loss of $2.3 \%$ for DMFS, and of $6.4 \%$ for OS, compared to an administration time close to the recommended 1 year. Thus, an entire course of administration ( 1 year), compared to less exposure time, could potentially prevent at least 6 deaths per 100 patients treated long-term. In fact, this exposure time was also significant in the analysis considering only patients who received any treatment with trastuzumab in relation to OS.

This study has some limitations, such as the long follow-up time, which implies changes in protocols. And despite the statistical design employed, the impact for a vast majority of patients who received no trastuzumab treatment $(95 \%$ and $81 \%$ in the $<63$ days and $<292$ days, respectively) groups makes it difficult to draw any correct conclusions about the effect of shorter exposure times. Despite the representativeness of our setting in a real-world public health service in a developing country, we cannot draw definitive conclusions on efficacy. However, even with significantly different groups, it was possible to observe, among those who received some amount of trastuzumab, that an optimal administration of treatment ( 1 year) is a greater protection factor than suboptimal treatments, according to current guidelines, making it inadvisable to shorten its administration.

\section{Conclusions}

The administration of trastuzumab improves survival in non-metastatic breast cancer patients, and is the main therapy for managing HER2positive overexpressing/amplified tumours. The entire course of trastuzumab administration achieves the maximum benefit, in contrast to the loss of protection for patients receiving suboptimal or no trastuzumab.

\section{Conflicts of interest}

The authors declared that there are no conflicts of interest.

\section{Funding statement}

The authors received no financial support for the research, authorship and/or publication of this article.

\section{Authors' contributions}

FACL conceived the study. FACL, ECM, CPN, LAM and PFRD designed the methodology and collected the data. FACL performed data analysis. RMA, RAR and MJBS supervised and validated data collection and analysis. FACL wrote the initial manuscript. All authors reviewed and approved the final draft.

\section{References}

1. Coates AS, Winer EP, Goldhirsch A, and Gelber RD, et al (2015) Tailoring therapies--improving the management of early breast cancer: St Gallen International Expert Consensus on the Primary Therapy of Early Breast Cancer 2015 Ann Oncol 26(8) 1533-1546

2. Goldhirsch A, Winer EP, and Coates AS, et al (2013) Personalizing the treatment of women with early breast cancer: highlights of the St Gallen International Expert Consensus on the Primary Therapy of Early Breast Cancer 2013 Ann Oncol 24(9) 2206-2223 
3. Rivenbark AG, O'Connor SM, and Coleman WB (2013) Molecular and cellular heterogeneity in breast cancer: challenges for personalized medicine Am J Pathol 183(4) 1113-1124

4. Hammond ME, Hayes DF, and Dowsett M, et al (2010) American Society of Clinical Oncology/College of American Pathologists guideline recommendations for immunohistochemical testing of estrogen and progesterone receptors in breast cancer (unabridged version) Arch Pathol Lab Med 134(7) e48-e72

5. Wolff AC, Hammond ME, and Hicks DG, et al (2013) Recommendations for human epidermal growth factor receptor 2 testing in breast cancer: American Society of Clinical Oncology/College of American Pathologists clinical practice guideline update J Clin Oncol 31(31) 3997-4013

6. Network TCGA (2012) Comprehensive molecular portraits of human breast tumours Nature 490(7418) 61-70

7. Engstrom MJ, Opdahl S, and Hagen Al, et al (2013) Molecular subtypes, histopathological grade and survival in a historic cohort of breast cancer patients Breast Cancer Res Treat 140(3):463-473

8. Ban M, Petric Mise B, and Vrdoljak E (2020) Early HER2-positive breast cancer: current treatment and novel approaches Breast Care (Basel) 15(6) 560-569

9. Garnock-Jones KP, Keating GM, and Scott $L$ (2010) Trastuzumab: a review of its use as adjuvant treatment in human epidermal growth factor receptor 2 (HER2)-positive early breast cancer Drugs 70(2) 215-239

10. Jensen MB, Nielsen TO, and Knoop AS, et al (2018) Mortality and recurrence rates among systemically untreated high risk breast cancer patients included in the DBCG 77 trials Acta Oncol 57(1) 135-140

11. Giuliano AE, Edge SB, and Hortobagyi GN (2018) Eighth edition of the AJCC cancer staging manual: breast cancer Ann Surg Oncol 25(7) 1783-1785

12. Hortobagyi GN, Connolly CE, and D'Orsi CJ, et al (2017) Breast AJCC Cancer Staging Manual 8th edn, eds MB Amin, S Edge, and F Greene, et al (New York: Springer) pp 589-636

13. Kaliks RA, Pontes Lde B, and Bognar CL, et al (2013) Treatment of breast cancer patients from a public healthcare system in a private center: costs of care for a pilot public-private partnership in oncology Einstein (Sao Paulo) 11(2) 216-223

14. Martins SJ and Yamamoto CA (2008) Clinical and economic issues in adjuvant chemotherapy for HER-2 positive breast cancer Rev Assoc Med Bras (1992) 54(6) 494-499

15. Edge SB, Byrd DR, and Compton CC, et al (2015) AJCC Cancer Staging Manual 7th edn (New York: Springer-Verlag)

16. Wyld L, Reed MWR, and Morgan J, et al (2021a) Bridging the age gap in breast cancer Impacts of omission of breast cancer surgery in older women with oestrogen receptor positive early breast cancer A risk stratified analysis of survival outcomes and quality of life Eur J Cancer 142 48-62

17. Wyld L, Reed MWR, and Collins K, et al (2021b) Bridging the age gap in breast cancer: cluster randomized trial of the effects of two decision support interventions for older women with operable breast cancer on quality of life, survival, decision quality, and treatment choices Br J Surg 108(5) 499-510

18. Mickey RM and Greenland S (1989) The impact of confounder selection criteria on effect estimation Am J Epidemiol 129(1) 125-137

19. Goldhirsch A, Wood WC, and Gelber RD, et al (2007) Progress and promise: highlights of the international expert consensus on the primary therapy of early breast cancer 2007 Ann Oncol 18(7) 1133-1144

20. Sun G, Jing H, and Wang S, et al (2021) Trastuzumab provides a comparable prognosis in patients with HER2-positive breast cancer to those with HER2-negative breast cancer: post hoc analyses of a randomized controlled trial of post-mastectomy hypofractionated radiotherapy Front Oncol 10605750 
21. Ministério Da Saúde B (2021) Portaria N 73, De 30 De Janeiro De 2013-inclui Procedimentos Na Tabela De Procedimentos, Medicamentos, Órteses/próteses E Materiais Especiais Do Sus E Estabelece Protocolo De Uso Do Trastuzumabe Na Quimioterapia Do Câncer De Mama Her-2 Positivo Inicial E Localmente Avançado [https://bvsms.saude.gov.br/bvs/saudelegis/sas/2013/prt0073_30_01_2013.html]

22. Moraes EL, Osorio-de-Castro CGS, and Caetano R (2016) Federal procurement of antineoplastic drugs in Brazil: analysis of imatinib mesylate, trastuzumab and L-asparaginase, 2004-2013 Physis Revista de Saúde Coletiva 26(4) 1357-1382

23. Pivot X, Romieu G, and Debled M, et al (2019) 6 months versus 12 months of adjuvant trastuzumab in early breast cancer (PHARE): final analysis of a multicentre, open-label, phase 3 randomised trial Lancet 393(10191) 2591-2598

24. Schneider BP, O'Neill A, and Shen F, et al (2015) Pilot trial of paclitaxel-trastuzumab adjuvant therapy for early stage breast cancer: a trial of the ECOG-ACRIN cancer research group (E2198) Br J Cancer 113(12) 1651-1657

25. Conte P, Frassoldati A, and Bisagni G, et al (2018) Nine weeks versus 1 year adjuvant trastuzumab in combination with chemotherapy: final results of the phase III randomized Short-HER studydouble dagger Ann Oncol 29(12) 2328-2333

26. Joensuu $\mathrm{H}$, Fraser J, and Wildiers $\mathrm{H}$, et al (2018) Effect of adjuvant trastuzumab for a duration of 9 weeks vs 1 year with concomitant chemotherapy for early human epidermal growth factor receptor 2-positive breast cancer: the SOLD randomized clinical trial JAMA Oncol 4(9) 1199-1206

27. Mavroudis D, Saloustros E, and Malamos N, et al (2015) Six versus 12 months of adjuvant trastuzumab in combination with dose-dense chemotherapy for women with HER2-positive breast cancer: a multicenter randomized study by the Hellenic Oncology Research Group (HORG) Ann Oncol 26(7) 1333-1340

28. Stewart P, Blanchette P, and Shah PS, et al (2020) Do all patients with HER2 positive breast cancer require one year of adjuvant trastuzumab? A systematic review and meta-analysis Breast 54 203-210 